\title{
A Comparative Analysis of Job Scheduling for Optimum Performance of Parallel Machines by Considering the Energy Consumption
}

\author{
Maurizio Faccio, Mojtaba Nedaei, and Francesco Pilati
}

\begin{abstract}
In this study, a comparative analysis of scheduling for machines and jobs was conducted by proposing a method, which aims to optimize the performance of the job-shop environment. A wide range of objective functions including make-span, maximum tardiness, total flow time, total tardiness, total weighted flow time, and total weighted tardiness, and energy consumption were considered. The decision variables of a manufacturing company including job's weight, starting and completion time, due date, releasing date, and processing time were also considered as inputs of the optimization model. Then, subject to the defined technical constraints of the system, a comparative analysis on the basis of eight scheduling methods was conducted to assess the performance indicators of the job-shop system. Results of the analysis showed the variations of different objective functions based on the proposed methods. The optimum make-span varied from minimum of 210 days estimated by the Earliest Due Date (EDD) method, up to 222 days estimated by the Critical Ratio (CR) method. Further recommendations were also made to optimize the involved decision variables in the considered job-shop environment. Results revealed that the maximum tardiness was best optimized using the EDD method, whilst the Shortest Processing Time (SPT) led to the best optimum value for the total flow time. Analysis of the energy evaluation of the machines showed that the optimum overall energy consumption has been observed with the value of 7,934 $\mathrm{kWh}$ for the first operating machine under the effect of the Minimum Slack method, whilst the optimum energy consumption for the second machine was observed with the value of 7,968 $\mathrm{kWh}$ using the First Come First Served (FCFS). Last stage of study recommended the optimum planning schedules and resource allocations of the jobs in the machines considering each scheduling method during the operation of the designed job-shop.
\end{abstract}

Index Terms-Scheduling; Performance Optimization; Parallel Machines; Jobs; Processing Machines; Manufacturing.

\section{INTRODUCTION}

In today's world, the scheduling, with no doubt, play a crucial role in managing the production processes, and optimizing the work schedules and workloads in production and manufacturing processes. It is noted that, the scheduling is contingent upon decision-making analysis and can be performed on a regular basis in several manufacturing firms,

Published on September 18, 2018

M. Faccio is with the Department of Management and Engineering, University of Padova, Vicenza, Italy (email: maurizio.faccio@unipd.it).

M. Nedaei is with the Department of Management and Engineering, University of Padova, Vicenza, Italy (email: mojtaba.nedaei@studenti.unipd.it)

F. Pilati is with the Department of Industrial Engineering, University of Bologna, Bologna, Italy (francesco.pilati3@unibo.it). and industrial services with the aim of investigating the assignment and issuance of resources to tasks over a considered time periods and its primary purpose is to enhance different objective functions. The resources and tasks in a manufacturing firm can be in several different forms. In this regard, the resources can be considered as machines in a workshop, runways at an airport, crews at a construction site, processing units in a computing environment, and so on. The tasks may be operations in a production process, take-offs and landings at an airport, stages in a construction project, executions of computer programs, and other related items. During the operation of the jobs and machines, every task has a designated priority level, an earliest possible starting time and a due date. In addition, the objectives can also be in several different forms. One goal may be towards minimizing the number of tasks processed subsequent to their corresponding due dates [1].

A scheduling process, which is performed properly would aid to enhance the efficiency and capacity utilization of the operating machines during their performance. In addition, the task times can be reduced, and the profitability for a designated product could be increased. Furthermore, the resource duplication would directly have a significant influence on short-term decision-making during the production scheduling [2].

One of the preliminary works concerning the subject of scheduling dates back to 1958, where Johnson [3] for the first time, addressed a two machine flow shop problem. With passage of some time, the first heuristic methods for optimization of make-span during the flow shop scheduling analysis were then introduced by Hundal and Rajgopal [4]. Dai et al. [5] developed an energy-efficient scheduling model for a flexible flow shop problem through employing an improved genetic-simulated annealing algorithm. Shabtay and Zofi [6] investigated single machine scheduling with controllable processing times and an unavailability period to optimize the make-span. The purpose was to determine a job schedule by which the make-span is minimized. A constant factor approximation algorithm as well as a fully polynomial time approximation scheme (FPTAS) for solving the optimization problem was developed in this study. Li et al. [7] studied parallel-machine scheduling with machine-dependent maintenance periodic recycles. Two mathematical programming models were presented to tackle small-sized instances. It was proven that the MLPT algorithm enhances the objective value with a less average relative error. $\mathrm{Wu}$ and $\mathrm{Che}$ [8] introduced a memetic differential evolution algorithm for energy-efficient 
parallel machine scheduling. The objective was to minimize both make-span and total energy consumption. Their obtained results showed that their proposed algorithm outperforms NSGA-II and SPEA-II. Liu et al. [9] studied the job shop scheduling with integration of four buffering constraints. It was shown that the proposed heuristic algorithm is satisfactory in order to address the job shop scheduling with combination of buffering constraints in real time.

Obviously, there are many research works in the literature review addressing the scheduling problems for operating machines in the flow-shop environments. These studies have mostly considered the make-span and energy consumption as their primary objective functions. They have also used one or two common method of optimization for performing the analysis. However, a structure, which takes into account a considerably broader range of objectives and scheduling methods in order to address the operation of machines in flow-shops has not been deeply investigated yet. The current research, different from what elaborated in the performed researches in the past, aims to design a job-shop environment under effect of different technical constraints, to optimize seven objectives of the system, which include make-span, maximum tardiness, total flow time, total tardiness, total weighted flow time, total weighted tardiness, and the energy consumption. Additionally, eight scheduling methods, including apparent tardiness cost with setups (ATCSs), earliest due date (EDD), minimum slack (MS), first come, first served (FCFS), longest processing time (LPT), shortest processing time (SPT), weighted shortest processing time (WSPT), and critical ratio (CR) were implemented to investigate the studied performance indicators of the job-shop system. The research also aims to compare the obtained results of the proposed scheduling methods and to suggest the most efficient ones for each objective function and finally to demonstrate the optimum planning schedules and the resource allocations of the jobs in the machines considering each scheduling method.

The contributions of the current paper are as follows:

(1) A manufacturing system has been simulated considering a data set of decision variables including job's weight, releasing date, due date, processing time, starting time, completion time, tardiness and weighted tardiness.18 jobs distributed over two processing machines were considered.

(2) A comparative analysis and study of scheduling of machines and jobs by proposing a new structure was perforemd with the purpose of optimizing the performance of the job-shop environment.

(3) A wide range of objective functions which have an effect on the operations and performance of the machines are considered and analyzed.

(4) The optimum values of the objective functions and the suitable resource allocation of the jobs in the system are also proposed.

The remainder of this research is organized to be as follows: in the second chapter of manuscript, the material and methods of the research are developed. Additionally, the objective functions, the data extracted from the considered manufacturing company, the scheduling methods, the governing equations, and the assumptions and constraints were explained in this part. In the next section of the research, after performing the optimization and calculations, the results are obtained and elaborated, and finally in the last section of the research, conclusions and future research directions were drawn and explained.

\section{MATERIAL AND METHODS}

In the first part of the methodology section, the scheduling goals of the manufacturing system are described. Then, the constraints of the model are explained and afterwards, the scheduling methods are proposed.

\section{A. Definition of the primary objective functions}

In order to perform a detailed analysis, seven objective functions are considered during the current analysis. The followings describe each studied objective functions.

\section{1) Make-span}

Make-span is a key criterion in a manufacturing system. It is defined as the time difference between the starting, and finishing of a sequence of jobs or tasks. In a manufacturing company, the purpose of the optimization would be to minimize the maximum make-span in a considered range of jobs [10].

\section{2) Tardiness $(T)$}

The tardiness is typically defined as a criteria of a delay, which can occur during the processing of the jobs in the operating machines. It can also be defined as the difference between the completion time and the due date of that job. In a flow-shop environment, one purpose of the optimization would be to minimize the maximum tardiness $\left(T_{\max }\right)$ to achieve better performance of the manufacturing system. In general, the tardiness in indicator of the due dates, and the maximum tardiness measures the worst violation of the due dates. The other indicators of the tardiness, which are the total tardiness $\left(\sum T_{j}\right)$ and the total weighted tardiness ( $\left.\sum W_{j} T_{j}\right)$ should also be considered in the analysis.

\section{3) Flow time}

This parameter is defined as the time when a job is completed minus the time the job was first available for processing. In this study, two indicators of flow time, which are total flow time $\left(\sum C_{j}\right)$, and total weighted flow time ( $\left.\sum W_{j} C_{j}\right)$ are considered as objective functions [1].

\section{4) Energy consumption (EC)}

Energy consumption of machines is one of the effective criteria involved during the analysis of the job processing machines. In this study, in addition to the aforementioned objective functions, the energy consumption of the machine has also been estimated based on the following equations (Eqs. 1 - 3 respectively) [10]:

$$
\begin{aligned}
& E C[k W h]=\left\{C_{j}-C_{1}-\sum_{j=2}^{n} P_{j}\right\} \times I P+M E_{c} \\
& M E_{c}[k W h]=\sum_{j=1}^{n} Y_{j}+P P \sum_{j=1}^{n} P_{j}
\end{aligned}
$$


$Y_{j}[k W h]=\left\{S E-\left\{\left(C_{j+1}-P_{j+1}\right)-C_{j}\right)\right\} I P$

Where $E C$ is the energy consumption $(k W h), M E_{C}$ is the machine's energy characteristics, $C_{j}$ is completion time for job $j(h), P_{j}$ is the processing time for job, $j(\mathrm{~h}) . I P$ is the Ideal Power $(\mathrm{kW}), \mathrm{Y}_{\mathrm{j}}$ is decision variable considering the energy for the setup $(k W h), S E$ is the energy setup $(k W)$, and $P P$ is the required power to process the job $(k W)$. The machine's energy characteristic, $M E_{C}$ is a decision variable of the system, which depends upon ideal power, setup energy and the power to process the jobs.

Furthermore, the following constraints were implemented for the considered operating machines:

$$
I P=11, P P=14, S E=18
$$

In addition, the considered system is assumed to be a flow shop environment consisting of two operating machines, which has interfering operation hours, with zero buffer capacity. Furthermore, owing to the requirements of the manufacturing company, it is also assumed that the second machine will have a much more operating hours to process the first and last jobs of the machine.

\section{B. Assumptions and constraints of the model}

In the current analysis, we have considered two parallel machines with a number of 18 jobs. The design parameters include decision variables for each job including the weight, the release date, the due date, processing time, starting and finishing date, tardiness, and the weighted tardiness. These parameters are extracted from the studied manufacturing company and shown in the Table 1 in the appendix. The unit of the decision variables are considered to be in days.

As shown in the above-mentioned table, $W_{j}$ is the job's weight, $R l s$. is the releasing date of the job, $S_{t}$ is the starting time of the job, $T$ is the tardiness, and $W_{t}$ is the weighted tardiness. The first stage of the analysis process has begun with the data collection including the design variables of the manufacturing system. Afterwards, the assumptions, constraints, limitations, and objective functions were defined and imposed through the optimization model. The job shop's status in the Table 1 (appendix) is either indicated with $A$, when the first machine is operating, or $B$, when the second machine is processing the jobs.

Based on the requirements of our manufacturing company, a series of assumptions and constraints are imposed prior to the optimization process. The followings explain the constraints applied during the analysis:

$\operatorname{Max}\left(d_{17}\right)=\operatorname{Max}\left(d_{18}\right)=32$

$W_{1}=W_{2}=W_{6}=1$

$P_{4}=P_{5}=P_{6}=32$

$P_{\text {min }}=12, P_{\text {max }}=40$

$P_{J B}>P_{J A}$ $d_{2}=d_{3}, d_{6}=d_{7}, d_{8}=d_{9}=d_{10}, d_{13}=d_{14}$

Where $d$ is the due date, $\operatorname{Max}\left(d_{j}\right)$ is indicative of the maximum amount of the due date, $W_{j}$ is the weight for the job, $j, P_{j}$ is the processing time for job, $j, P_{\min }$ is the minimum processing time, $P_{\max }$ is the maximum processing time, $P_{j B}$ is the processing time job $j$ for when the machine's status, $B$ (or $A$ ) is defined. In the next section, the scheduling methods, which are utilized during the optimization process are explained, and the objective functions were evaluated. Scheduling analysis of the jobs together with the flow of coming jobs to the flow-shop environment were the last stage of the process.

\section{The proposed scheduling methods}

In the current research, eight heuristics were proposed to estimate the objective functions during the scheduling analysis and optimization process. The following subsections explain the aforementioned methods [1]:

\section{1) Apparent tardiness cost with setups (ATCSs)}

The ATCS method is as a result of integrating different methods simultaneously. It calculates the index of job, $j$ at time, $t$ when job $l$ has completed its processing on the machine as follows:

$$
I_{j}(t, l)=\frac{W_{j}}{P_{j}} \exp \left(-\frac{\max \left(d_{j}-P_{j}-t, 0\right)}{K_{1} \bar{p}}\right) \exp \left(-\frac{S_{l j}}{K_{2} \bar{S}}\right)
$$

Where $\bar{S}$ is the average value of the setup times for the considered jobs, which are remained to be scheduled, $\bar{p}$ is the average processing time, $d_{j}$ is the due date of the job, $\mathrm{j}$, $S_{i j}$ is the setup times of the jobs $j$ and $l, W_{j}$ is the weight of the job, $\mathrm{p}_{j}$ is processing time of job, $j, K_{l}$, the due date related scaling parameter, and $\mathrm{K}_{2}$ is the setup time factor, which is related to a scaling parameter. The scaling parameters are dimensionless values and they're independent of the units utilized to express various quantities. The two scaling parameters, $\mathrm{K}_{1}$ and $\mathrm{K}_{2}$ can be regarded as functions. During the current analysis, the coefficients of the ATCSs method, i.e., $K_{1}$ and $K_{2}$ are considered to be 8 and 14 respectively. It is noted that the optimization using this method considers the jobs subject to sequence dependent setup times.

\section{2) Critical ratio $(C R)$}

This method is considered as one of the most typical scheduling methods during the analysis to aid the most production scheduling systems. Being an index number, the $\mathrm{CR}$ can be determined through dividing the remaining time until the due date by the work time remaining.

Once the CR is estimated for each work station, the priority order would be given through using the value of the estimated critical ratio. The priority order is performed from smaller to larger values.

\section{3) The earliest due date (EDD)}

The EDD method is a common dispatching scheduling method, which relies on the scheduling of the due dates. The EDD is considered as a priority rule, which sequences the jobs in a queue on the basis of their (operation or job) due 
dates.

\section{4) First come, first served (FCFS)}

This method is defined as a method, which aims to perform the sequencing of the jobs, on the basis that they first appear to the queue of the jobs.

\section{5) Longest processing time (LPT)}

The LPT performs the sequencing of the job flows on the basis of the longest processing time. This rule would typically be assigned at $t=0$ the $\mathrm{m}$ longest jobs to the $\mathrm{m}$ machines. Afterwards, whenever a machine is freed the longest job among those not yet processed is to put on the machine. This heuristic method attempts to place the shorter jobs more towards the end of the schedule, where they can be utilized for balancing the loads.

\section{6) Minimum Slack (MS) method}

The MS method, which is on the basis of the EDD, is a dynamic scheduling rule with the purpose of ordering a designated number of jobs in machine according to max $\left(D_{j}\right.$

$\left.-P_{j}-t, 0\right)$, which is time dependent. This implies that at some point in time job $j$ may have a higher priority than job $k$ and at some later point in time jobs $j$ and $k$ may have the same priority.

\section{7) Shortest processing time (SPT)}

The SPT method is defined as a common scheduling dispatching rule, which aims to perform the sequencing of the coming jobs to the machine on the basis of shortening the processing time of the jobs.

\section{8) Weighted shortest processing time (WSPT)}

The WSPT method is defined as a common scheduling dispatching rule, which aims to perform the sequencing of the coming jobs to the machine on the basis of shortening the processing time of the jobs. Among all studied rules, the EDD and WSPT are those of the dispatching rules, which are applied in a single or parallel machine environments. However, they can also be applied in a more complicated machine environment such as the flexible flow shop (FFS) and the flexible job shop (FJS). They are therefore applied in the following way: each time a machine is freed, then the system checks out which jobs should keep operating on the machine. The system then uses the following data for the priority rules: the due date of a candidate job is then the due date at which the job has to leave the system. The processing time, which is plugged in the WSPT rule is the sum of the processing times of all the remaining operations of that job.

\section{RESULTS AND DISCUSSION}

The current analysis has been performed for two parallel machines with 18 jobs. Subsequently, the results explaining the estimated values for the objective functions have been obtained based on each scheduling rule.

One important part of the study was to analyze the energy consumption (EC) of the processing machines. The EC criteria plays a big part in the reliability of a designed job/flow-shop. The less EC values, the more efficient that job/flow-shop would become. The overall variations of energy consumption during the current analysis based on the proposed methods are indicated in the Table 1I in the main text. The minimum EC has occurred with the value of 7,934 $k W h$ during operation of the first machine using the MS method, whilst for the second operating machine, the minimum EC was 7,968 $k W h$ when the FCFS method is implemented. The maximum EC has occurred with the value of $12,370 \mathrm{kWh}$ during the operation of first machine using the CR method, whereas for the second machine, the EC values ranged from minimum of 7,968 $\mathrm{kWh}$ up to maximum of $10,688 \mathrm{kWh}$.

Table 2 in the appendix section reveals the overall evaluation of the primary objective functions (except the energy consumption) under effect of each scheduling method. Looking through the table, it is evident that the different scheduling methods have led to different values of the objective functions. When comparing the results of the objective functions using each scheduling method, it was concluded that the make-span and the maximum tardiness were among those objective functions, which had resulted in closer estimated values in terms of the employed method of scheduling. The most significant difference concerning the estimated values of the objective functions was observed for the total weighted tardiness $\left(\sum W_{j} T_{j}\right)$ followed by the total tardiness $\left(\sum T_{j}\right)$, and the total flow time $\left(\sum C_{j}\right)$. The analysis shows that the make-span has varied from minimum of 210 days to maximum of 222 days. The EDD method together with the LPT have resulted in optimum level of the make-span with value of 210 days. The EDD rule was also considered as the only rule, among the studied heuristics, which was able to minimize the maximum tardiness with value of 178 days. The SPT rule was able to optimize the total flow time together with the total tardiness with the values of 1898 , and 1452 days respectively. Results of the study have been generalized so that for any number of the job s, and machines, the considered objective functions can be estimated under specified technical constraints.

The ATCS and WSPT were able to best optimize the total weighted completion time and tardiness. To sum up, the current analysis gives a great sense of creating a compressive scheduling and planning method, which recommends the optimum values of the most important objective functions in a manufacturing system that should be considered from the design phase of the job/flow-shop till during the operation of the processing machines. It also makes comparisons of the different scenarios on the basis of the proposed scheduling rules.

Overall, taking into account the evaluation of the proposed scheduling methods, and the investigated objective functions, and considering an average criterion of the optimized values, it has become evident that the EDD rule together with the SPT, in most cases, had a great performance in the optimization of the scheduling parameters as well as the energy consumption of the processing machines.

It is worthwhile to mention that analyzing the resource allocation and the distribution of the processed jobs during the operation of machines is a crucial aspect of understanding their performance, and operation process, since it can provide a great perspective with regard to the flow of coming jobs, which are processed, as well as their 
processing time among all studied jobs, with consideration of different sequencing methods. Relations 8 -11 depict the order of the jobs on the basis of the scheduling methods, i.e. EDD and SPT, which proved to have a better performance compared with other scheduling methods. The processing hours and the machine number are considered. These relations also aim to demonstrate the optimum planning schedules and the resource allocations of the jobs in the machines considering each scheduling method. Overall, it can be concluded that the EDD and SPT rules have produced a similar pattern of the job's distributions, though, after the sixth job in the first machine and the $14^{\text {th }}$ job in the second machine, there has been some minor differences in the order of the coming jobs to the job-shop environment. The maximum finishing hours, have occurred in the second machine, with the value of 210 and 212 days on the basis of the EDD, and SPT rules respectively.

Aside from the energy consumption of machines together with the completion time, two effective parameters during the analysis of the machine scheduling are the make-span and tardiness. As a matter of fact, minimizing the make-span plays a crucial role in enhancing the efficiency of a manufacturing system, since if the make-span is not decreased as much as possible, the order of the products will not be shipped on time. On the other hand, in order to decrease the lateness of performing the tasks, minimizing the tardiness parameter is also another primary goal to achieve. Thus, in the following diagram (as shown in the Fig. 1), with consideration of each scheduling method, a trade-off between these two parameters has been performed, which concludes that, for the majority of the scheduling methods, there is a direct relation between the make-span and the maximum tardiness in terms of the time. In other words, as the maximum tardiness increases, the make-span increases. The only two methods, by which the objective functions had experienced a sudden decrease were the ATCS and the LPT.

TABLE I: THE OVERALL ENERGY CONSUMPTION VALUES BASED ON EACH SCHEDULING METHOD

\begin{tabular}{ccc}
\multicolumn{3}{c}{ SCHEDULING METHOD } \\
\hline \hline Scheduling method & $\begin{array}{c}\text { Overall EC } \\
\left(M_{1}\right)(k W h)\end{array}$ & $\begin{array}{c}\text { Overall EC } \\
\left(M_{1}\right)(k W h)\end{array}$ \\
\hline ATCS $(8,14)$ & 8,216 & 8,550 \\
CR & 12,370 & 10,688 \\
EDD & 8,044 & 8,396 \\
FCFS & 8,494 & 7,968 \\
LPT & 8,250 & 8,904 \\
MS & 7,934 & 8,286 \\
SPT & 7,986 & 8,430 \\
WSPT & 8,578 & 8,790 \\
\hline \hline
\end{tabular}

Sequencing based on the EDD rule:

$$
\begin{aligned}
& M_{1}=J_{1}-J_{10}-J_{12}-J_{13}-J_{5}-J_{6}-J_{16}-J_{17}-J_{9} \\
& M_{2}=J_{2}-J_{11}-J_{3}-J_{4}-J_{14}-J_{15}-J_{17}-J_{1}-J_{18}
\end{aligned}
$$

Sequencing based on the SPT rule

$$
\begin{aligned}
& M_{1}=J_{1}-J_{10}-J_{12}-J_{13}-J_{5}-J_{6}-J_{8}-J_{16}-J_{17} \\
& M_{2}=J_{2}-J_{11}-J_{3}-J_{4}-J_{14}-J_{7}-J_{9}-J_{15}-J_{18}
\end{aligned}
$$

\section{CONCLUSIONS AND Future RESEARCH}

In the current study, a comparative analysis of scheduling and optimization of parallel machines in a job-shop environment through applying several advanced scheduling methods and objective functions is conducted. Results revealed the most efficient decision variables, as well as the relationships among the objective functions and the studied scheduling methods. Optimization results showed that the EDD method followed by the FCFS had better efficiency compared with other methods in obtaining the optimum make-span. The EDD was also able to demonstrate a great performance to minimize the total flow time, total tardiness, and maximum tardiness in the designed flow shop.

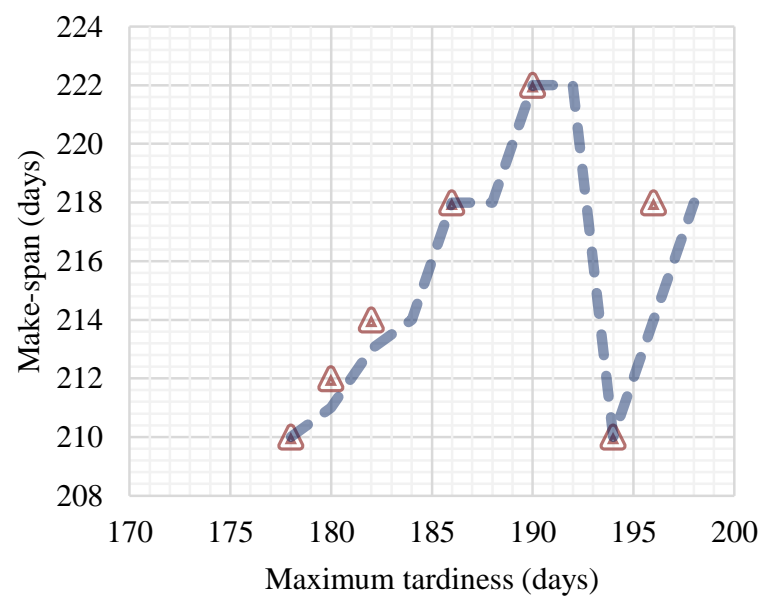

Fig. 1. The trade-off evaluation between the selected objective functions.

In addition, the ATCS was considered as the most efficient method for minimizing the total weighted flow time, and the total weighted tardiness. Subsequently, the total flow time together with the total tardiness were best optimized using the SPT rule.

Analysis of the energy consumption was also another aspect of the research which showed how much each machine and jobs have consumed energy. This matter is particularly important when measuring the energy performance of a designed flow shop. It was concluded that the MS method has led to highest maximum EC during the operation of first machine. On the other hand, the FCFS for the first machine has caused less EC negative energy balance. It also became clear that the energy consumption of the machines was significantly dependent upon the completion time, and processing time of the jobs.

In the last section of the paper, the flow distribution of the processed jobs during the operation of machines was studied. It became evident that the first operating machine had more processing hours when the CR, FCFS, LPT, and MS methods are applied during the scheduling process, whilst for the second machine, ATCS, EDD, SPT, and WSPT led to finishing the operation in a longer span of time. 
Future work concerning the current research aims to investigate the scheduling of the machines in a flow shop environment through considering variations in the scheduling parameters such as the two coefficients of the ATCS optimization method, together with the sample input data. Another matter would be to investigate the current methodology by applying other frameworks such as flexible flow shops, job-shops, flexible job-shops and other environments by which machines and jobs would be operating under designated constraints and design variables of the system.

\section{APPENDIX}

In this part of the paper, Tables 1 and 2 of the paper are demonstrated. The related explanations are provided in the section 1 and 3 of the manuscript.

TABLE. 1. THE DATA EXTRACTED FROM THE STUDIED MANUFACTURING COMPANY

\begin{tabular}{cccccccccc}
\hline \hline Job No. & $W_{j}$ & $R l s$. & $\begin{array}{c}\text { Due } \\
\text { date }\end{array}$ & $P(t)$ & Status & $S_{t}$ & $C_{j}$ & $T$ & $W_{t}$ \\
\hline J1 & 1 & 8 & 15 & 12 & A & 186 & 115 & 184 & 184 \\
J2 & 1 & 10 & 18 & 14 & A & 198 & 144 & 194 & 194 \\
J3 & 2 & 13 & 18 & 18 & A & 126 & 148 & 122 & 244 \\
J4 & 2 & 15 & 19 & 32 & B & 142 & 169 & 139 & 278 \\
J5 & 2 & 17 & 20 & 32 & B & 158 & 169 & 156 & 312 \\
J6 & 1 & 11 & 22 & 22 & A & 198 & 151 & 196 & 196 \\
J7 & 4 & 20 & 22 & 33 & B & 122 & 161 & 120 & 480 \\
J8 & 2 & 11 & 25 & 24 & A & 164 & 154 & 161 & 322 \\
J9 & 2 & 11 & 25 & 25 & A & 176 & 155 & 173 & 346 \\
J10 & 8 & 22 & 25 & 35 & B & 100 & 159 & 97 & 776 \\
J11 & 3 & 12 & 27 & 26 & A & 140 & 157 & 137 & 411 \\
J12 & 7 & 14 & 29 & 27 & A & 102 & 161 & 97 & 485 \\
J13 & 7 & 18 & 32 & 28 & A & 18 & 163 & 10 & 70 \\
J14 & 9 & 20 & 32 & 29 & A & 20 & 165 & 12 & 108 \\
J15 & 10 & 25 & 27 & 32 & B & 74 & 163 & 73 & 730 \\
J16 & 12 & 26 & 28 & 34 & B & 74 & 167 & 74 & 888 \\
J17 & 14 & 26 & 32 & 38 & B & 44 & 170 & 43 & 602 \\
J18 & 17 & 26 & 32 & 40 & B & 42 & 173 & 22 & 714 \\
\hline \hline
\end{tabular}

TABLE. 2. THE ESTIMATED OBJECTIVE FUNCTIONS OF THE JOB-SHOP ENVIRONMENT.

\begin{tabular}{ccccccc}
\hline \hline $\begin{array}{c}\text { Scheduling } \\
\text { method }\end{array}$ & $\mathrm{C}_{\max }$ & $\mathrm{T}_{\max }$ & $\sum C_{j}$ & $\sum T_{j}$ & $\sum W_{j} C_{j}$ & $\sum W_{j} T_{j}$ \\
\hline ATCS (8, 14) & 218 & 196 & 2476 & 2030 & 10218 & 7340 \\
CR & 222 & 190 & 2071 & 1625 & 16284 & 13406 \\
EDD & 210 & 178 & 1912 & 1466 & 15182 & 12304 \\
FCFS & 218 & 186 & 1958 & 1512 & 15716 & 12838 \\
LPT & 210 & 194 & 2416 & 1970 & 10730 & 7852 \\
MS & 214 & 182 & 2221 & 1775 & 11914 & 9036 \\
SPT & 212 & 180 & 1898 & 1452 & 15940 & 12612 \\
WSPT & 218 & 196 & 2476 & 2030 & 10218 & 7340 \\
\hline \hline
\end{tabular}

\section{REFERENCES}

[1] M.L. Pinedo, Scheduling: Theory, Algorithms, And System, New York: Springer, 2008, ISBN 978-1-4614-2361-4

[2] A. Azzi, M. Faccio, A. Persona, \& F. Sgarbossa, "Lot splitting scheduling procedure for make-span reduction and machine capacity increase in a hybrid flow shop with batch production", The International Journal of Advanced Manufacturing Technology, vol. 59(5), pp. 775-786, 2012

[3] S.M. Johnson, "Optimal two and three-stage production schedules with set-up times included", Naval Research Logistics Quarterly, vol. 1, pp. 61-68, 1954.

[4] T.S. Hundal, \& J. Rajgopal, "An extension of palmer's heuristic for the flow-shop scheduling problem", International Journal of Production Research, vol. 26(6), pp. 1119-1124, 1988.

[5] M. Dai, D. Tang, A. Giret, M.A. Salido, and W.D. Li, "Energyefficient scheduling for a flexible flow shop using an improved genetic-simulated annealing algorithm", Robotics and ComputerIntegrated Manufacturing, vol. 29(5), pp. 418-429, 2013.
[6] D Shabtay, \& M. Zofi, "Single machine scheduling with controllable processing times and an unavailability period to minimize the makespan", International Journal of Production Economics, vol. 198, pp 191-200, 2018.

[7] G. Li, M. Liu, S.P. Sethi, \& D. Xu, "Parallel-machine scheduling with machine-dependent maintenance periodic recycles", International Journal of Production Economics, vol. 186, pp.1-7, 2017.

[8] X. Wu, \& A. Che, "A memetic differential evolution algorithm for energy-efficient parallel machine scheduling", Omega, Available online in corrected proof: https://doi.org/10.1016/j.omega.2018.01.001, 2018.

[9] S.Q. Liu, E. Kozan, M. Masoud, Y. Zhang, \& F.T. Chan, "Job shop scheduling with a combination of four buffering constraints", International Journal of Production Research, vol.56(9), pp.32743293, 2018 .

[10] G. Mouzon, M.B. Yildirim, \& J. Twomey, "Operational methods for minimization of energy consumption of manufacturing equipment", International Journal of Production Research, vol.45(18-19), pp. 4247-4271, 2017. 